\title{
Spleen Function and Anxiety in Chinese Medicine: A Western Medicine Perspective
}

\author{
Yin Kwan Chung, Jihang Chen, Kam Ming Ko* \\ Division of Life Science, Hong Kong University of Science \& Technology Clear Water Bay, Hong Kong, China \\ Email: *bcrko@ust.hk
}

How to cite this paper: Chung, Y.K., Chen, J.H. and Ko, K.M. (2016) Spleen Function and Anxiety in Chinese Medicine: A Western Medicine Perspective. Chinese Medicine, 7, 110-123.

http://dx.doi.org/10.4236/cm.2016.73012

Received: June 21, 2016

Accepted: August 22, 2016

Published: August 26, 2016

Copyright $\odot 2016$ by authors and Scientific Research Publishing Inc. This work is licensed under the Creative Commons Attribution International License (CC BY 4.0)

http://creativecommons.org/licenses/by/4.0/

\section{(c) (i) Open Access}

\begin{abstract}
This article aims to illustrate the function of the Spleen in Traditional Chinese Medicine (TCM) in the context of modern medicine. TCM has been practiced since ancient times in China for the prevention and/or treatment of diseases. Yet, the complete understanding of its theoretical basis in relation to clinical practice from the modern medicine perspective is still lacking. According to TCM theory, the Spleen, as one of the five Zang (i.e., visceral organs), plays an important role in various physiological functions, including digestion and absorption of nutrients, regulation of water retention and excretion, facilitation of blood perfusion to skeletal muscle and on the optimal functioning of the immune system. Clinical applications of herbal formulations for the treatment of Spleen dysfunction (i.e., Spleen deficiency-a decline in Spleen function) and their pharmacological activities are described. The view point of TCM on how emotions (or Qing Zhi) can influence the body function is introduced. The relationship between anxiety and Spleen function has been analyzed by reviewing relevant research studies in modern medicine. These findings suggest that the cause/consequence relationship between anxiety and Spleen function may be bi-directional.
\end{abstract}

\section{Keywords}

TCM Theory, Spleen Function, Anxiety

\section{Introduction}

TCM is a medical practice originating from ancient China through thousand years of experiential use. It still plays an important role in medical care in China and Southeast Asian countries despite the advent of Western medicine. However, the practice of TCM has become increasingly popular in western countries because of its sophisticated and successful treatments for many health conditions and even diseases that are regarded as 
untreatable or incurable by conventional Western medicine. As TCM and Western medicine represent two distinct medical approaches with different origins, both diagnostic strategies and interpretations of symptoms of diseases in TCM are quite different from those of Western medicine. As the mystery goes, TCM provides an "unscientific" but yet "successful" approach to medical practice. It has therefore attracted the efforts of many researchers in deciphering the "science" behind TCM. This will be instrumental in the acceptance and promotion of the practice of TCM in the global arena for the betterment of mankind.

According to TCM theory, the human body consists of five Zang and six Fu (i.e., all visceral organs), which are characterized by the five elements, namely Metal, Wood, Water, Fire and Earth, in the realm of the "Five Element" theory. The five Zang (i.e. visceral organs) are functionally interlinked with each other by generating and restricting mode of action, resulting in an optimally functioning body by maintaining a holistic Yin-Yang balance. Disease will develop when this balance is distorted, as in the cases of over-restricting and counter-restricting (i.e., insulting). Furthermore, the $\mathrm{Zang}$ - $\mathrm{Fu}$ (i.e. all visceral organs) interplay responds to environmental conditions, and these conditions-referred to as "external evils", can distort the Yin-Yang balance and result in disease.

In this article, we focus on understanding the concept of dampness, one of the "external evils" commonly occurring in individuals living in areas with high humidity. According to TCM theory, exposure to humid weather conditions can induce dampness in the body. Water associated with dampness can upset the function of the Spleen, one of the Zang (i.e. visceral organs) with Earth characters, as Water acts by insulting the Earth [1]. Thus, prior to investigating what pathophysiological conditions are influenced by dampness, an understanding of the function of the Spleen in TCM is essential. The functions of the Spleen in relation to TCM theory will first be reviewed. Symptoms of Spleen dysfunction (i.e., Spleen deficiency-a decline in Spleen function) and their treatments by TCM herbal formulations will then be described, with pharmacological activities of the formulations being illustrated in terms of Western medicine. Finally, an interesting concept in TCM concerning how anxiety might impair Spleen function will be discussed.

\section{Functions of Spleen}

TCM theory purports that there are three aspects of Spleen function (Figure 1). Firstly, the Spleen regulates the transport and metabolism of water and nutrients in the body. Stomach, the associated $\mathrm{Fu}$ (i.e. organs) of the Spleen, works to digest ingested food into various components, which are transported to the Spleen for distribution throughout different parts of the body. Water absorbed will be transported upward to the Lung for the generation of $Q i$ (energy/nutrients), and excessive water and metabolic wastes produced by different regions of the body are transported downward to the Kidney for excretion [1]. The accumulation of "humor", as in the case of edema or phlegm formation, 


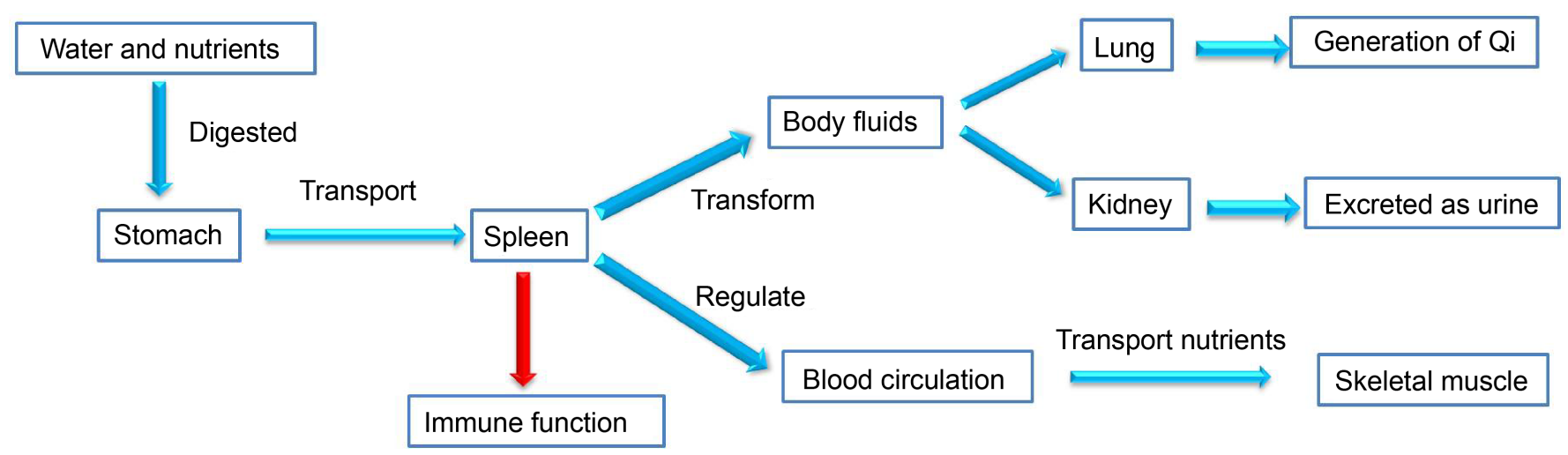

Figure 1. The functions of Spleen in TCM. The Spleen works with the Stomach to regulate the transport and metabolism of water and nutrients. Water absorbed is transported upward to the Lungs for the generation of $Q i$, and excessive water plus metabolic wastes produced by different regions of the body are transported downward to the Kidney for excretion. In addition, the $S p l e e n$ regulates the circulation of blood. The transport of nutrients in blood to skeletal muscle is controlled by the Spleen. Therefore, Spleen appears to be an important determinant for maintaining a proper functioning of skeletal muscle. The Spleen is also related to immune function.

is regarded as a pathological outcome of Spleen dysfunction. Spleen dysfunction can also lead to a malfunctioning of food digestion and absorption, or even a pathophysiological condition known as "metabolic syndrome" [2]. A study has shown that Glutinous Rice, which is regarded as a functional food for strengthening Spleen function, improves digestive function in rats with Spleen dysfunction. The beneficial effect of Glutinous Rice on digestive function is associated with increases in levels of salivary amylase and plasma gastrin and motilin, as well as a decrease in plasma level of somatostatin [3]. Experimental findings suggest that the Spleen is functionally related to digestive processes in Western medicine. Also, a rat model of Spleen dysfunction associated with dampness-heat was found to have the over-expression of aquaporin, a water channel commonly found in the lungs, digestive system and kidney, suggesting that the function of the Spleen may be related to the functioning of these systems in regulating water content in the body [4].

Secondly, the Spleen regulates the circulation of blood. Spleen functions to enable the flow of blood within blood vessels in the right direction and then deliver nutrients ( $Q i)$ throughout the body. Thus, sub-cutaneous bruising and hemorrhage, such as conditions associated with hematuria and excessive menstrual bleeding, are caused by Spleen malfunctioning [1]. From the Western medicine point of view, the prevention of internal bruising is regarded as a function of the blood/circulatory system, through the regulation of blood clotting and capillary permeability, with the latter preventing the leakage of red blood cells into the extracellular space. Research has shown that patients with Spleen dysfunction generally show an abnormal morphology of blood platelets, leading to a decrease in their ability to aggregate and release clotting factors. Thrombocytes in Spleen dysfunction patients have been found to exhibit a shorter lifespan, resulting in a higher likelihood of hemorrhage. Spleen dysfunction patients were also found to have an increased capillary fragility, such that that they are more prone to capillary rupture, leading to internal bruising. 
In connection with the functions of nutrient metabolism and transport as well blood circulation, the Spleen appears to be an important determinant for the maintenance of proper functioning of skeletal muscle. This is because the transport of nutrients in blood to skeletal muscle is controlled by the Spleen. This is corroborated by the experimental observations that both the configuration and function of extensor muscle were significantly deteriorated in mice with Spleen dysfunction [5], and that Spleen dysfunction patients have higher blood lactate levels, as a result of anaerobic glycolysis in skeletal muscles [6]. These observations may well explain why tiredness and muscle pain are commonly observed in patients with Spleen dysfunction.

Thirdly, the Spleen is related to immune function. TCM theory states that one will not be influenced by "external evils" if the Spleen is functioning optimally. The Spleen, being endowed with the Earth nature, is thought to be the center of other elements. If the Spleen functions well, other Zang (i.e. visceral organs) will also be better off [1]. Results from a number of studies have established the functional relationship between the Spleen and the immune system. Patients with Type 2 Diabetes Mellitus with Spleen dysfunction showed an obvious immune dysfunction (i.e., either hypo- or hyper-activity of immune cells [7]. Patients suffering from ulcerative colitis with Spleen/Kidney Yang deficiency showed a reduction of serum Treg and TGF $\beta 1$ levels, indicative of an impairment in immune tolerance [8]. Cang Zhu (Atractylodis Chinensis Rhizoma), a Spleen-invigorating herb, has been found to improve the immune function of rats with Spleen dysfunction as well as protect and repair damaged mucosal tissues [9].

\section{TCM Approach to the Treatment of Spleen Deficiency}

The effectiveness of various TCM herbal formulations for the treatment of various patterns of Spleen dysfunction has been well established [10], but the underlying pharmacological mechanisms are relatively unclear. Gui Pi Tang (Restore the Spleen Decoction) and Huang Tu Tang (Yellow Earth Decoction), which are two commonly prescribed preparations for the treatment of Spleen dysfunction, have been investigated for their pharmacological properties [11].

Herbal components in the two formulations act mainly on the digestive system. The main constituent herb in Gui Pi Tang (Restore the Spleen Decoction) namely Huang Qi (Astragali Sinesis Radix), has been found to reduce the secretion of gastric juice and suppress the activity of pepsin by reducing the secretion of gastrin, thereby preventing the development of ulcers [12]. Bai Zhu (Atractylodes Macrocephala Rhizoma) protects gastric mucosa against gastric acid-induced injury, and it also suppresses abnormal peristaltic motion in the small intestine [13] [14]. Gan Cao (Glycyrrhiza Uralensis Radix) suppresses the secretion of gastric juice and reduces the acidity of gastric juice by neutralizing the hydrochloric acid [15]-[17]. The main herbal constituent of Huang $\mathrm{Tu}$ Tang (Yellow Earth Decoction), namely, Zao Xin Tu / Fu Long Gan (Ignited Yellow Earth; Terra Flava Usta), is rich in aluminum oxide, which protects the gastric mucosa by neutralizing acidic gastric juice [18]-[20]. Zhi Fu Zi (Aconiti Lateralis Preparata Radix) in Huang Tu Tang (Yellow Earth Decoction) was found to suppress gastric emp- 
tying, and protect against gastric ulceration [21]-[23]. Bai Zhu and Gan Cao in the preparation also produced significant protection to the digestive system [13]-[17].

Gui Pi Tang (Restore the Spleen Decoction) and Huang Tu Tang (Yellow Earth Decoction) contain medicinal herbs that can suppress hemorrhage and facilitate blood flow. Huang Qi in Gui Pi Tang was found to prevent thrombus formation in blood vessels by inhibiting the action of phosphodiesterase. Huang $Q i$ also improves the microcirculation and reduces the viscosity of blood [24]. Huang Q $i$ and the "assistant" herb, Ginseng (Panax Ginseng Radix), can dilate blood vessels and promote a smooth flow of blood. Ginsenoside Rg1 and Rg3 in Ginseng were found to decrease blood viscosity and prevent the aggregation of thrombocytes [25] [26]. Zao Xin Tu (Terra Flava Usta) in Huang Tu Tang (Yellow Earth Decoction) is calcium-rich, and its "assistant" herb, Donkey-hide Gelatin (Er Jiao; Corii Asini Colla), is rich in glycine that can promote the uptake of calcium, an essential factor in blood clotting (i.e., preventing internal bleeding). Donkey-hide Gelatin also reduces blood clotting time, and increases the number of plasma thrombocytes [27]. Zhi Fu Zi in Huang Tu Tang also promotes the agglutination of thrombocytes [21]-[23]. It was observed that opposing pharmacological actions are produced by Gui Pi Tang (Restore the Spleen Decoction) and Huang Tu Tang (Yellow Earth Decoction), with the former reducing blood clot formation and thus smoothening blood flow, and the latter facilitating blood clot formation and preventing internal bleeding. Yet, both formulations are effective in treating Spleen dysfunction [11] [21].

With respect to the enhancement of immune function, Huang Qi in Gui Pi Tang (Restore the Spleen Decoction) contains Astragalus polysaccharides that can boost immunity by inhibiting the action of suppressive T-cells and thereby increase the activities of other types of T-cells, as well as stimulating the growth of plasma cells for the production of antibodies. Various herbal components in the formulation regulate the production of immunoglobulins and increase the activity of natural killer cells. Huang $Q i$ has been found to induce the $\gamma$-interferons and thereby boost the activity of natural killer cells [28]. Ginsenosides from Ginseng increases the concentration of IgG, IgA, IgM and IL-2 in plasma, and stimulates the activity of natural killer cells and other types of white blood cells [25] [26] [29]. Guang Pi Tang (Restore the Spleen Decoction) was found to be effective in suppressing the production of autoantibodies in patients with chronic immune thrombocytopenic purpura, an autoimmune disease characterized by a reduction in the number of thrombocytes [30]. Donkey-hide Gelatin in Huang Tu Tang (Yellow Earth Decoction) was found to increase the phagocytotic activity of monocytes and the activity of natural killer cells. Bai Zhu stimulates the production of antibodies and the activity of phagocytes by increasing the production of IL-1 and IL-2 from lymphocytes. Baicalin in Huang Qin (Scutellariae Baicalensis Radix) increases the amount of cAMP in monocytes, thereby promoting the differentiation of lymphocytes. Gan Cao can induce the production of interferons that can activate natural killer cells. Gan Cao suppresses the immune response by decreasing the concentration of antigens, and glycyrrhinic acid in Gan Cao was found to enhance the produc- 
tion of IL-1 and immunoglobulins [31] [32]. Taken together, herbal formulations for treating Spleen dysfunction also possess immunomodulatory activity.

\section{Anxiety and Spleen Function}

The practice of TCM adopts a holistic approach in making diagnosis of diseases, with the categorization of symptoms into various patterns of Zang-fu (i.e. visceral organs) disorders. Furthermore, TCM views that various emotions, or Qing Zhi, do influence an individual's physiological functions.

In TCM theory, there are seven Qing-five of which are collectively called $Z h i$. The five $Z$ hi, namely, joy, anger, grief, anxiety and fright, are characterized by five elements, namely, Fire, Wood, Metal, Earth and Water, respectively. As Zang-fu (i.e. all visceral organs) are linked to these five elements, the over-expression of each $Z$ hi can affect the function of its respective Zang-fu (i.e. all visceral organs). In light of the Five Element theory, all $Z$ hi are related by generating, restricting, over-restricting and reverse-restricting one another (Figure 2). Thus, balancing the expression of the five Zhi is thought to be crucial in maintaining psychological and physical health [33].

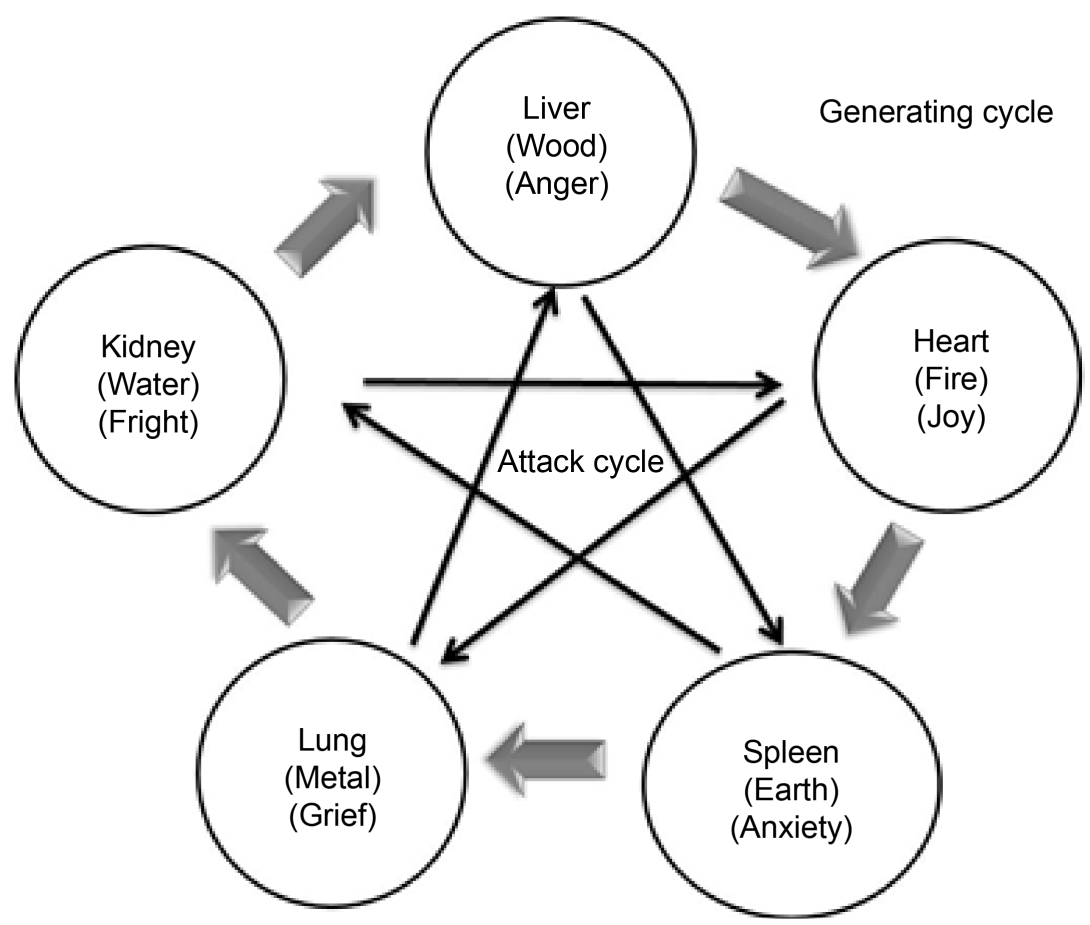

Figure 2. Relationship between Zang-fu and Five Zhi in TCM. Five Zhi, namely, joy, anger, grief, anxiety and fright, are characterized by five elements, namely, Fire, Wood, Metal, Earth and Water, respectively. As Zang-fu are linked to the five elements, the over-expression of each Zhi can affect the function of its respective Zang-fu. In addition, there is a generating cycle and restricting (or attack) cycle in which the five Zang-fu interact. The generating cycle describes the ways in which each element, serving as a mother, promotes the growth and development of the following child element. The restricting cycle provides for a check and balance system among all of the elements. The third form of interaction, namely insulting (not shown in the figure), describes the triggering of the attack. Thus, the direction of the insulting is the reverse of the restricting cycle. 
Both Spleen and anxiety belong to the Earth, giving rise to a TCM theory stating that "Anxiety impairs Spleen". According to TCM theory, over-focusing or over-thinking on one subject will lead to anxiety, with resultant dysfunction in the transport of water and nutrients around the body and the stagnation of $Q i$ (energy/ nutrients), indicative of a poorly functioning of the Spleen. Zhi arises as a phenotype of an outcome from one's thoughts, and prolonged anxiety can worsen the functions of other Zang (i.e. visceral organs), which is consistent with the inter-functional relationship among various Zang (i.e. visceral organs) [33]. Interestingly, anger can be used to alleviate anxiety. Anger belongs to Wood, which restricts the Earth according to the Five Element theory. Thus, by inducing a state of rage, the effects of anger can effectively alleviate a state of anxiety [33]. Although such psychotherapy is theoretically sound, little is currently known about the scientific basis of such treatment.

It is worth noting the difference between stress and anxiety. Stress can be defined as a short-term psychological pressure when only limited resources are available to finish a task, whereas anxiety can be defined as a long-term worry about something uncertain in the future. Yet, a prolonged state of stress can also lead to anxiety. Modern biomedical research has shown a causal relationship between anxiety and Spleen-regulated function, but the physiological characteristic of anxiety should first be defined prior to achieving an understanding of such an interrelationship. Studies have shown that General Anxiety Disorder (GAD) can be characterized by an asymmetrical expression of the Sympathetic Nervous System (SNS) and the Hypothalamic-Pituitary-Adrenal (HPA) axis [34]. Based on this observation, it can be deduced that the influence of anxiety on Spleen function is likely mediated by both nervous and endocrine factors. The SNS, a component of the autonomic nervous system, serves to inhibit digestive functions (including peristalsis) and the enteric nervous system, an intrinsic nervous system in the digestive process. The HPA axis regulates a series of endocrine glands, wherein the hypothalamus secretes Corticotropin Releasing Hormone ( $\mathrm{CRH}$ ) that stimulates the pituitary gland to secrete adrenocorticotropic hormone (ACTH), and ACTH in turn stimulates the secretion of cortisol from the adrenal cortex (Figure 3).

Cortisol primarily activates gluconeogenesis and the metabolism of fat, protein and carbohydrate in cells. However, the effect of immunosuppression by cortisol explains the functional relationship between HPA axis and the Spleen, with the latter being a regulator of immune function. The secretion of cortisol, which is stimulated by cytokines, can inhibit the further production of cytokines by immunosuppression, and this negative feedback loop of regulation is primarily used to prevent the over-activation of the immune system [35]. However, cortisol, as a "stress" hormone, has been found to be strongly associated with anxiety. The overproduction of cortisol under conditions of anxiety leads to immunosuppression, a symptom of Spleen dysfunction in TCM. Other studies have found that GAD patients have a lower level of peripheral benzodiazepine receptors on their $\mathrm{T}$ lymphocytes [36], suggesting that benzodiazepines (which are antianxiety drugs) may also exert a regulatory effect on immune function [37].

The correlation between anxiety and gastrointestinal (GI) diseases has been studied. 


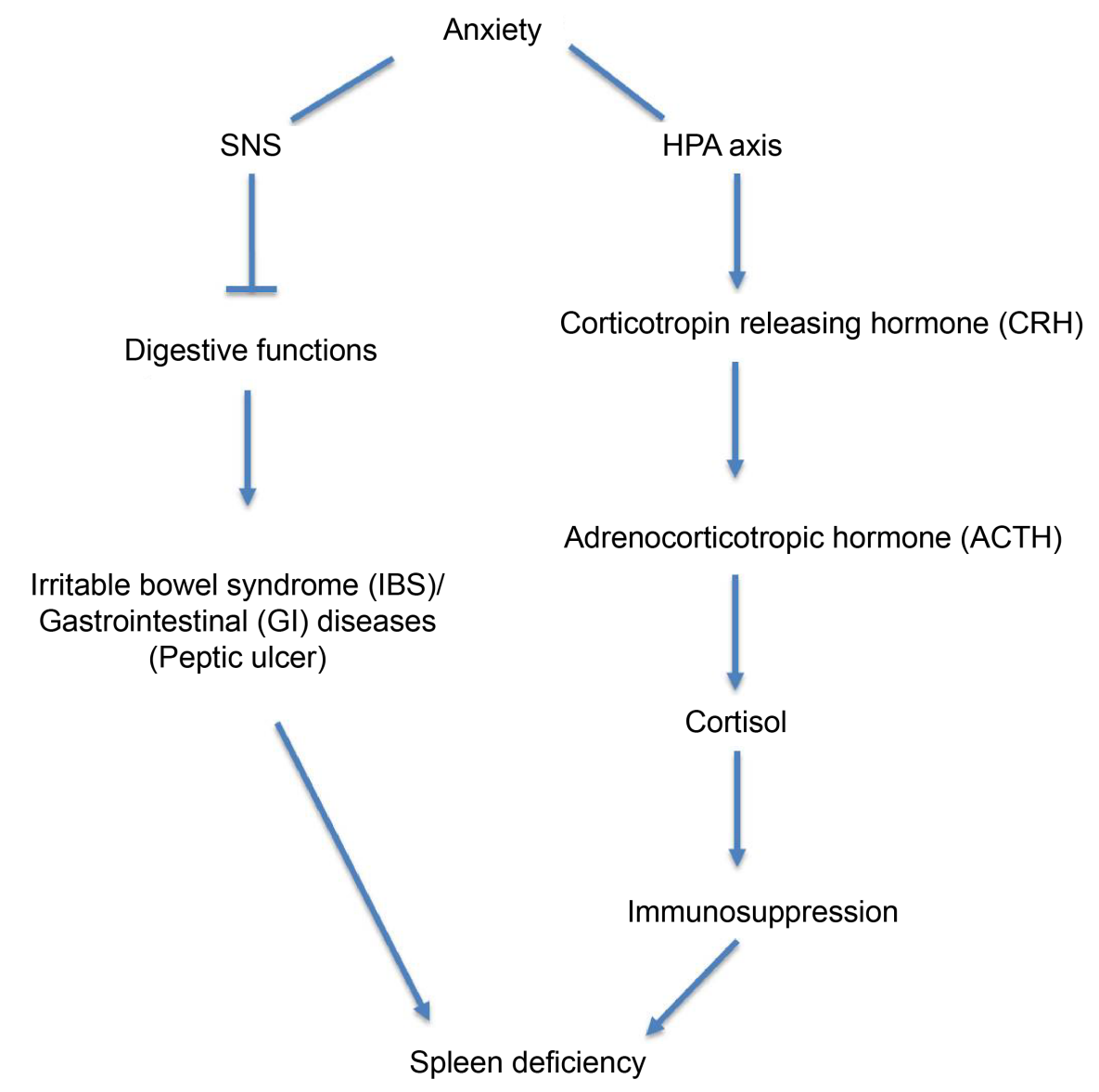

Figure 3. Anxiety and Spleen deficiency. In Western medicine, general anxiety disorder (GAD) can be characterized by the asymmetrical expression between the sympathetic nervous system (SNS) and the hypothalamal-pituitary-adrenal (HPA) axis. The SNS serves to inhibit digestive functions. The over-stimulation or under-stimulation of the digestive system by SNS, leads to contradicting irritable bowel (IBS) symptoms and gastrointestinal (GI) diseases, which are associated with Spleen dysfuction in TCM. On the other hand, the HPA axis regulates a series of endocrine glands, wherein the hypothalamus secretes corticotropin releasing hormone $(\mathrm{CRH})$ that stimulates the pituitary gland to secrete adrenocorticotropic hormone (ACTH), and ACTH in turn stimulates the secretion of cortisol from the adrenal cortex, with resultant immunosuppression and thus Spleen dysfuction. The secretion of cortisol, which is also stimulated by cytokines, can inhibit the further production of cytokines. This negative feedback loop of regulation is primarily used to prevent the over-activation of the immune system.

It was found that Irritable Bowel Syndrome (IBS) was closely associated with anxiety [38]. IBS is a collection of patterns of altered bowel movement in the absence of any damage in GI tract [39]. This may be explained by the over-stimulation or under-stimulation of the digestive system by the SNS, leading to contradicting IBS symptoms such as constipation and diarrhea. A study also shows that stressors can disrupt the function of GI microbiota by inducing a neural response, which increases the risk of mucosal infection and inflammation. Stress on the GI mucosal immune system can lead to the translocation of pathogenic microbes from the GI tract to the interior of the body, with the resultant infection and the associated inflammatory response [40]. Se- 
vere and sustained anxiety can induce peptic ulceration. Symptoms of peptic ulcer, including digestive discomfort and bleeding along the digestive tract, can be related with Spleen dysfunction, in which the Spleen fails to sustain the function of the digestive system and regulate the circulation of blood.

A causal relationship between anxiety and peptic ulceration is supported by clinical and experimental observations. It has been shown, for example, that stress, which is correlated to anxiety, can cause peptic ulcer in absence of Helicobacter pylori [40]. An increase in the number of patients with peptic ulcers that are not caused by bacteria or non-steroidal anti-inflammatory drugs was observed after the traumatic earthquake in East Japan on 11 March, 2011, suggesting that psychological stress arising from this natural disaster can be a significant factor in causing peptic ulcers [41]. "Executive monkey" studies have also shown that animals that had been exposed to stressors were more prone to developing gastric ulcers [42].

The causal relationship between psychological stress and peptic ulceration can be explained by the adverse effect of the SNS innervation of the mucosa and Gut-Associated Lymphoid Tissue (GALT). Sympathetic nerve fibers enter the alimentary canal along arteries and eventually terminate on blood vessels in the close vicinity of the mucosa. As such, mucus secretoneurone is regulated by inhibitory signals via $\alpha 2$ adrenergic signaling with noradrenaline as the neurotransmitter. Sympathetic noradrenergic nerve fibers also innervate the interdomal region of the submucosa with T-lymphocytes and plasma cells [43]. While the mechanism by which over-innervation of the SNS can produce an immunomodulatory action remains unclear, a hypothesis concerning the development of peptic ulcers caused by anxiety can be postulated based on the relationship between the SNS and gastrointestinal mucosa. Since anxiety can be characterized by the over-stimulation of the SNS [44], overwhelming signals from the SNS may trigger a higher output of inhibitory signals that suppress the production or release of secretoneurone. This leads to a decreased production of mucus, resulting in erosion of the mucosa and muscle layer of the duodenum by gastric juice.

In TCM, it is believed that anxiety arises from an imbalance of Zang (i.e. visceral organs) functions, wherein different combinations of functional imbalances among Zang (i.e. visceral organs) result in various symptoms. Yet, as the Five Element theory goes, Earth, which is considered as the center of the five elements, will eventually be hindered by the imbalance of other elements. Thus, the treatment of different types of anxiety is primarily focused on invigorating the function of the Spleen [44]. Gui Pi Tang (Restore the Spleen Decoction), which is a commonly used formulation in treating anxiety, not only ameliorates the symptoms of Spleen dysfunction caused by anxiety, but also directly alleviates anxiety. From the perspective of TCM theory, Gui Pi Tang (Restore the Spleen Decoction) mobilizes the stagnant $Q i$ (energy/nutrients) and nourishes shen in the Heart, which is thought to be the master of Qing Zhi. As anxiety is pathologicallyrelated to the stagnation of $Q i$, a smooth flow of $Q i$ will ease anxiety [44]. The pharmacological properties of Gui Pi Tang (Restore the Spleen Decoction) have been investigated. In treating peptic ulcers, Dang Gui (Angelicae Sinensis Radix) can prevent 
bleeding in the alimentary canal caused by peptic ulceration. Huang Qi and Ginseng were found to be effective in preventing peptic ulcer development [12]-[21]. It was also observed that Gan Cao produced an anti-ulcer action by increasing gastric mucosal defensive factors [45]. Gan Cao also promotes the proliferation of gastric mucosal cells and protects the mucosa by releasing endogenous prostaglandins that can stimulate mucus production. On the other hand, Huang Qi, Gan Cao and Suan Zao Ren (Zizyphi Spinosae Semen) were found to produce a tranquillizing action [10] [11] [46]-[48].

On the basis of TCM theory, a causal relationship between anxiety and Spleen function has been clearly established. However, a recent study has shown that the status of GI microbiota can in turn induce anxiety-like behavior [49]. The complete removal of GI microbiota leads to a decrease in anxiety-like behavior and alteration in the neurochemistry of the central nervous system [50]. Such an interaction, known as the microbiome-gut-brain axis, is poorly understood in molecular and physiological terms, but the finding does suggest the possibility that the correlation between anxiety and Spleen function can be bi-directional. In this connection, patients with Spleen dysfunction show a higher blood lactate level [6], which is consistent with the symptoms of tiredness and muscle fatigue in Spleen dysfunction. While patients with GAD exhibited significantly higher blood lactate levels than those of control subjects after vigorous exercise (indicative of Spleen dysfunction), anxiety attacks could be induced by the infusion of lactate into controls, suggesting that Spleen dysfunction may also be a cause of anxiety [51].

The practice of TCM remains popular in Asian countries despite the advent of Western medicine. While TCM and Western medicine belong to two distinct treatment systems, the explanation of TCM theory in the context of Western medicine can hopefully bridge the theoretical gap between TCM and Western medicine and thus facilitate the integration of the two therapeutic approaches to the prevention and/or treatment of diseases. In this article, we have endeavored to describe the function of the Spleen in TCM using the language of Western medicine. Hopefully, concerted efforts of this type can serve to modernize the theory of TCM which can be comprehended by individuals living in the $21^{\text {st }}$ century.

\section{References}

[1] Wang, S.L. (1990) Clinical Study on the Spleen and Stomach Theory. People's Medical Publishing House, Beijing.

[2] Liu, X.X. and Yan, S. (2015) Intervention Effect of Traditional Chinese Medicine Yi Tang Kang on Metabolic Syndrome of Spleen Deficiency. Asian Pacific Journal of Tropical Medicine, 8, 162-168. http://dx.doi.org/10.1016/S1995-7645(14)60309-6

[3] Zhang, H., Wang, J., Liu, Y. and Sun, B. (2015) Glutinous Rice Amylopectin Can Adjust the Plasma Gut-Regulated Peptide Levels in Rhubarb-Induced Spleen Deficiency Rats. Food \& Function, 7, 938-942. http://dx.doi.org/10.1039/C5FO00859J

[4] Li, Z.H., Wang, J., and Cai, L.L. (2011) A Review on Research Progress of Relationship between Diseases Caused by Dampness and Aquaporin. Journal of Chinese Integrative Medicine, 9, 5-10. http://dx.doi.org/10.3736/jcim20110102 
[5] Zhang, Q.W., Li, G.Y., Ren, Y.P. and Gao, Y.F. (2015) Effect of Two Pi Deficiency Syndrome Models on the Configuration and Function of the Skeletal Muscle in Mice. Chinese Journal of Integrated Traditional and Western Medicine, 35, 71-75.

[6] Zheng, X.F., Tian, J.S., Liu, P., Xing, J. and Qin, X.M. (2014) Analysis of the Restorative Effect of Bu-Zhong-Yi-Qi-Tang in the Spleen-Qi Deficiency Rat Model Using (1) H-NMRBased Metabonomics. Journal of Ethnopharmacology, 151, 912-920.

http://dx.doi.org/10.1016/j.jep.2013.12.001

[7] Chen, L., Yang, Z., Chen, W., Li, R., Lin, C., Guan, L., Zhu, Z., Chen, R., Li, S., Zhao, L., Zeng, J. and Wang, J. (2015) Differential Expression of Immune-Related Genes between Healthy Volunteers and Type 2 Patients with Spleen-Deficiency Pattern. Journal of Traditional Chinese Medicine, 35, 646-652. http://dx.doi.org/10.1016/S0254-6272(15)30154-0

[8] Yang, G., Liu, L, He, X., et al. (2014) The Th17/Treg Immune Balance in Ulcerative Colitis Patients with Two Different Chinese Syndromes: Dampness-Heat in Large Intestine and Spleen and Kidney Yang Deficiency Syndrome. Evidence-Based Complementary and Alternative Medicine, 2015, Article ID: 264317.

[9] Liu, F., Liu, Y. and Tian, C. (2015) Effect of Rhizoma Atractylodis Extract in Protecting Gastric Mucosa and Modulating Gastrointestinal Immune Function in a Rat Model of Spleen Deficiency. Journal of Southern Medical University, 35, 343-347.

[10] Effertha, T., Shan, L. and Zhang, Z.W. (2016) Tonic Herbs and Herbal Mixtures in Chinese Medicine. World Journal of Traditional Chinese Medicine, 2, 10-25. http://dx.doi.org/10.15806/j.issn.2311-8571.2015.0037

[11] Flaws, B. and Sionneau, P. (2001) The Treatment of Modern Western Medical Diseases with Chinese Medicine: A Textbook \& Clinical Manual.Blue Poppy Enterprises, Inc., Boulder.

[12] Yang, B., Ji, X., Cui, N. and Wang, S. (2015) Effect of Different Component of Astragalus Polysaccharides on the Gastrointestinal Motility of the Model Rats with Dampness Stagnancy due to Spleen Deficiency. Journal of Nanjing University of Traditional Chinese Medicine, 4, 372-375.

[13] Xu, Q., Wang, J.H., Wang, R.J., Liu, X.Q., Chen, Z.H. and Wu, Y.L. (2003) Effect of Buzhong Yiqi Decoction on Gastrin Receptor Combination and Its Protective Mechanism for Gastric Mucosa Damage in Spleen-Deficiency Rats. Journal of the Guangzhou University of Traditional Chinese Medicine, 20, 51-56.

[14] Wang, C.C. (2006) Study on the Processing of Atractylodis Rhizoma. Yearbook of Chinese Medicine and Pharmacy, 24, 121-156.

[15] Filimban, W., ElSawy, N., Header, E.A. and El-Boshy, M. (2015) Evaluation of Aqueous Extract of Salvadora persica and Glycyrrhiza glabra in Treatment of Gastric Ulcer. Jökull Journal, 65, 275-293.

[16] Awaada, A.S., El-Meligya, R.M. and Solimanb, G.A. (2013) Natural Products in Treatment of Ulcerative Colitis and Peptic Ulcer. Journal of Saudi Chemical Society, 17, 101-124. http://dx.doi.org/10.1016/j.jscs.2012.03.002

[17] Ahmad, B.A., Rao, M.U.S., Muhammad, A., Zin, T., Mohamad, N.H., Mohamad, N. and Mohd, K.S. (2014) Reviews of Herbal and Their Secondary Metabolites in the Treatment of Ulcerative Colitis and Peptic Ulcer. Journal of Applied Pharmaceutical Science, 4, 80-90.

[18] Wagner, H., Bauer, R., Melchart, D., Xiao, P.G. and Staudinger, A. (2011) Chromatographic Fingerprint Analysis of Herbal Medicines: Thin-Layer and High Performance Liquid Chromatography of Chinese Drugs. Volume 2, Springer, Berlin. http://dx.doi.org/10.1007/978-3-7091-0763-8

[19] Rong, Z. (2008) Frequently Used Nomenclature and Aliases of the Minerals, Chemical 
Formula Use in Taoist Alchemy. Hong Dao, 37, 48-52.

[20] Duan, S. (2011) Retention-Enema Treatment of Severe Antibiotic Associated Diarrhea: A Clinical Analysis. Chinese Journal of Nosocomiology, 21, 1654-1656.

[21] Chen, J.K. (2004) Chinese Medical Herbology and Pharmacology. Art of Medicine Press, Williams.

[22] Yu, Q., Luan, Y., Li, X. and Sun, R. (2012) Research Development on Efficacy of Prepared Aconite Root Related to Chemical Material Basis and Pharmacological Effects. Chinese Journal of Pharmacovigilance, 9, 674-678.

[23] Ao, H. and Cao, X. (2014) Comparison of Recognition on the Toxicity of Fuzi in the Ancient and Modern Times. Chinese Journal of Library and Information Science for Traditional Chinese Medicine, 38, 57-60.

[24] Ma, H.X. and Zhang, Z.X. (2004) Progress in the Pharmacological Researches on Astragalus membranaceus. Journal of Shaanxi College of Traditional Chinese Medicine, 27, 73-75.

[25] Liu, J., Pei, M., Zheng, C., Li, Y., Wang, Y., Lu, A. and Yang, L. (2013) A Systems-Pharmacology Analysis of Herbal Medicines Used in Health Improvement Treatment: Predicting Potential New Drugs and Targets. Evidence-Based Complementary and Alternative Medicine, 2013, Article ID: 938764. http://dx.doi.org/10.1155/2013/938764

[26] Wang, Y. (2001) Progress in the Pharmacological Researches on Ginseng. Ginseng Research, 13, 2-9.

[27] Wang, D., Ru, W., Xu, Y., Zhang, J., He, X., Fan, G., Mao, B., Zhou, X. and Qin, Y. (2014) Chemical Constituents and Bioactivities of Colla corii asini. Drug Discoveries \& Therapeutics, 8, 201-207. http://dx.doi.org/10.5582/ddt.2014.01038

[28] Yang, Z. and Wang, Q. (2013) Progress of Basic and Clinical Study on Immunization of APS. World Chinese Medicine, 8, 833-836.

[29] Kang, S. and Min, H. (2012) Ginseng, the "Immunity Boost": The Effects of Panax ginseng on Immune System. Journal of Ginseng Research, 36, 354-368.

http://dx.doi.org/10.5142/jgr.2012.36.4.354

[30] Yamaguchi, K., Kido, H., Kawakatsu, T., Fukuroi, T., Suzuki, M., Yanabu, M., Nomura, S., Kokawa, T. and Yasunaga, K. (1993) Effects of Kami-Kihi-to (Jia-Wei-Gui-Pi-Tang) on Autoantibodies in Patients with Chronic Immune Thrombocytopenic Purpura. The American Journal of Chinese Medicine, 21, 251-255. http://dx.doi.org/10.1142/S0192415X93000297

[31] Gao, H., Shao, S. and Wang, G. (2004) Research Progress of Glycyrrhiza Uralensis Fisch. Journal of Jinggangshan Medical College, 11, 8-11.

[32] Qiao, H. and Ma, T. (1992) Protective Effect of Glycyrrhiza uralensis Fish(Lx) on Anaphylactic Shock in Rats Sensitized with Penicilloyl-Protein. Journal of Zhengzhou University (Science Medical), 27, 14-17.

[33] Suo, Y.C. (1986) Novel Spleen and Stomach Theory. Shanxi Science and Technology Publishing House, Taiyuan.

[34] Reeves, J.W., Fisher, A.J., Newman, M.G. and Granger, D.A. (2016) Sympathetic and Hypothalamic-Pituitary-Adrenal Asymmetry in Generalized Anxiety Disorder. Psychophysiology, 53, 951-957. http://dx.doi.org/10.1111/psyp.12634

[35] Besedovsky, H., Chrousos, G. and Rey, A.D. (2008) The Hypothalamus-Pituitary- Adrenal Axis. Academic, Amsterdam.

[36] Rocca, P., Beoni, A.M., Eva, C., Ferrero, P., Zanalda, E. and Ravizza, L. (1998) Peripheral Benzodiazepine Receptor Messenger RNA Is Decreased in Lymphocytes of Generalized An- 
xiety Disorder Patients. Biological Psychiatry, 43, 767-773.

http://dx.doi.org/10.1016/S0006-3223(97)00279-5

[37] Shigeru, M., Takuya, M., Tohru, N., Masaharu, T. and Masahiko, S. (1998) Peripheral Type Benzodiazepine Receptor in T Lymphocyte Rich Preparation. Life Sciences, 63, 1423-1430. http://dx.doi.org/10.1016/S0024-3205(98)00409-3

[38] Lee, S., Wu, J., Ma, Y.L., Tsang, A., Guo, W.J. and Sung, J. (2009) Irritable Bowel Syndrome Is Strongly Associated with Generalized Anxiety Disorder: A Community Study. Alimentary Pharmacology \& Therapeutics, 30, 643-651. http://dx.doi.org/10.1111/j.1365-2036.2009.04074.x

[39] Grundmann, O. and Yoon, S.L. (2010) Irritable Bowel Syndrome: Epidemiology, Diagnosis, and Treatment: An Update for Health-Care Practitioners. Journal of Gastroenterology and Hepatology, 25, 691-699. http://dx.doi.org/10.1111/j.1440-1746.2009.06120.x

[40] Herszényi, L., Juhász, M., Mihály, E. and Tulassay, Z. (2015) Peptic Ulcer Disease and Stress. Orvosi Hetilap, 156, 1426-1429. http://dx.doi.org/10.1556/650.2015.30249

[41] Kanno, T., Iijima, K., Koike, T. and Shimosegawa, T. (2015) Relationship between Disaster Stress and Peptic Ulcers. Nihon Rinsho. Japanese Journal of Clinical Medicine, 73, 12091214.

[42] Murison, R. (2000) Gastric Ulceration. Encyclopedia of Stress, Volume 3, Academic Press, Cambridge.

[43] Straub, R.H., Wiest, R., Strauch, U.G., Härle, P. and Schölmerich, J. (2006) The Role of the Sympathetic Nervous System in Intestinal Inflammation. Gut, 55, 1640-1649. http://dx.doi.org/10.1136/gut.2006.091322

[44] Yeung, W.F., Chung, K.F., Ng, K.Y., Yu, Y.M.B., Zhang, S.P., Ng, B.F.L. and Ziea, E.T.C. (2015) Prescription of Chinese Herbal Medicine in Pattern-Based Traditional Chinese Medicine Treatment for Depression: A Systematic Review. Evidence-Based Complementary and Alternative Medicine, 2015, Article ID: 160189. http://dx.doi.org/10.1155/2015/160189

[45] Ghader, J.A., Vahid, N., Ehsan, A., Mostafa, M. and Hadi, K. (2015) Antiulcer Properties of Glycyrrhiza glabra L. Extract on Experimental Models of Gastric Ulcer in Mice. Iranian Journal of Pharmaceutical Research, 14, 1163-1170.

[46] Chen, C.J., Li, M., Wang X.L. and Yuan, H. (2011) Effect of Sour Date (Semen ziziphi spinosae) Seed Extract on Treating Insomnia and Anxiety. In: Preedy, V.V., Watson, R.R. and Vinood, P., Eds., Nuts and Seeds in Health and Disease Prevention, Academic Press, Cambridge, 1037-1043.

[47] Liu, L., Liu, C., Wang, Y., Wang, P., Li, Y. and Lia, B. (2015) Herbal Medicine for Anxiety, Depression and Insomnia. Current Neuropharmacology, 13, 481-493. http://dx.doi.org/10.2174/1570159X1304150831122734

[48] Lee, K.H., Morris-Natschke, S., Qian, K., Dong, Y., Yang, X., Zhou, T., Belding, E., Wu, S.F., Wada, K. and Akiyama, T. (2012) Recent Progress of Research on Herbal Products Used in Traditional Chinese Medicine: The Herbs Belonging to the Divine Husbandman's Herbal Foundation Canon (Shén Nóng Běn Căo Jīng). Journal of Traditional and Complementary Medicine, 2, 6-26. http://dx.doi.org/10.1016/S2225-4110(16)30066-9

[49] Bailey, M.T. (2014) influence of Stressor-Induced Nervous System Activation on the Intestinal Microbiota and the Importance for Immunomodulation. Advances in Experimental Medicine and Biology, 817, 255-276. http://dx.doi.org/10.1007/978-1-4939-0897-4_12

[50] Cryan, J.F. and O’Mahony, S.M. (2011) The Microbiome-Gut-Brain Axis: From Bowel to Behaviour. Neurogastroenterology and Motility, 23, 187-192.

http://dx.doi.org/10.1111/j.1365-2982.2010.01664.x 
[51] Kelly, D., Mitchell-Heggs, N. and Sherman, D. (1971) Anxiety and the Effects of Sodium Lactate Assessed Clinically and Physiologically. The British Journal of Psychiatry, 119, 129141. http://dx.doi.org/10.1192/bjp.119.549.129

Submit or recommend next manuscript to SCIRP and we will provide best service for you:

Accepting pre-submission inquiries through Email, Facebook, LinkedIn, Twitter, etc. A wide selection of journals (inclusive of 9 subjects, more than 200 journals)

Providing 24-hour high-quality service

User-friendly online submission system

Fair and swift peer-review system

Efficient typesetting and proofreading procedure

Display of the result of downloads and visits, as well as the number of cited articles

Maximum dissemination of your research work

Submit your manuscript at: http://papersubmission.scirp.org/ 